\title{
Caracterización morfológica, biomasa aérea y calidad en distintas poblaciones de Trichloris crinita
}

\author{
Gil Báez, C. '; Ordinola Agüero, R. ' ; Ernst, R.D.' y Ruiz, M.A. ${ }^{1,2 @ ~}$
}

'Facultad de Ciencias Exactas y Naturales. Universidad Nacional de La Pampa. La Pampa. Argentina. ${ }^{2}$ INTA, EEA Anguil Ing. Agr. Guillermo Covas. La Pampa. Argentina.

\section{Palabras ClaVe adiciOnales}

Choridoideae.

Pastizales.

Forraje.

\section{RESUMEN}

Gran parte de la superficie del territorio de Argentina se encuentra cubierta por pastizales naturales, siendo éstos aptos para el uso ganadero. Sin embargo, dichos ecosistemas han perdido biodiversidad y productividad a causa del uso antrópico, fundamentalmente debido al desplazamiento de las actividades ganaderas hacia áreas marginales presentando importantes restricciones productivas, lo cual acentúa la fragilidad de estos ambientes. Trichloris crinita (Lag.) Parodi, es una especie clave de manejo en los pastizales naturales de las regiones del Caldenal y del Monte; por lo tanto, es considerada apta para ser utilizada en procesos de rehabilitación, debido a que ha evolucionado en este tipo de ambientes. El objetivo de este trabajo fue caracterizar y evaluar en campo el comportamiento agronómico de 13 poblaciones de Trichloris crinita. En invernáculo se evaluó la germinación de las semillas, altura y número de hojas de las plantas. El transplante a campo se realizó cuando las plantas alcanzaron el estado de 4-5 hojas. Las determinaciones comenzaron a realizarse a los 8 meses del transplante; se hicieron recuentos de número de panojas, altura de planta y de dosel, diámetro de mata, biomasa aérea y calidad del forraje (proteína, fibra y digestibilidad). Se encontraron poblaciones de mayor biomasa, lo cual también coincidió con macollamiento y diámetro de mata. Si bien en este trabajo no se midió la producción de semilla, se pudo observar una mayor cantidad de inflorescencias en algunas poblaciones, entre ellas las del Caldenal y Ecotono Caldenal-Monte. Una de las poblaciones mostró muy baja persistencia luego de ser cortada, este fenotipo no es apto para pastoreos. En general las poblaciones presentaron calidad de forraje similar a otras especies estivales. Una de las poblaciones (Catamarca) además de su alta biomasa aérea, también mostró buena calidad de forraje. De acuerdo a los resultados obtenidos, se puede concluir que existe variabilidad entre las poblaciones de Trichloris crinita estudiadas, lo que permitirá seleccionar de acuerdo a su importancia agronómica, teniendo en cuenta fundamentalmente la biomasa total, la calidad del forraje, la producción de semillas y su persistencia bajo corte.

\section{Morphological characterization, aereal biomass and quality in different populations of Trichloris crinita}

\section{SUMMARY}

A large part of Argentina, is covered with natural grasslands, suitable for livestock use. However, those ecosystems have lost biodiversity and productivity due to anthropogenic use, caused mainly by displacement of livestock activities toward marginal areas, showing major production constraints and pointing out the weakness of these environments. Trichloris crinita (Lag.) Parodi, is a key species for rangeland management in Caldenal and Monte Regions. Therefore it is considered suitable to be used in reclamation processes, because it has also developed in these environments. The objective of this work was to characterize and evaluate the agronomic performance of 13 populations of Trichloris crinita in the field. Furthermore, seed germination, height and number of plant leaves were evaluated in the greenhouse. Transplanting was carried out when the plants reached the 4-5 leaf state. Determinations began to be performed at 8 months of transplantation; recounts of number of panicles, plant height, canopy height, plant diameter, number of tillers, biomass and forage quality (CP, DMD and NDF) were made. Populations with higher biomass were found, agreeing with tillering and plant diameter. Even though in this work seed production was not measured; it was possible to observe a greater number of inflorescences in some populations, including those of Caldenal and Ecotono Caldenal-Monte. One of the populations showed very low persistence after cutting; this phenotype is not suitable for grazing. Populations in general showed forage quality similar to other summer species. One of the populations (Catamarca), in addition to its aerial high biomass, also showed a good quality of forage. According to the results, it was concluded that there is variability among the populations studied, wich will be selected according to their agronomic importance, considering mainly the total biomass, quality, seed setting and its persistence under cutting. 


\section{INTRODUCCIÓN}

En Argentina, los pastizales ocupan el área húmeda, subhúmeda, semiárida y árida no cultivada, lo que comprende el 88,8 \% de la superficie del país (Nazar Anchorena, 1988). La mayoría de los pastizales naturales de Argentina se encuentran en las zonas áridas y semiáridas y son el sustento principal de la ganadería de cría (Nazar Anchorena, 1988). En muchos casos, como mencionan Morici et al. (2003; 2009) y Díaz (2007) estos pastizales naturales se encuentran empobrecidos por el mal uso al que han sido sometidos, reflejado en un reemplazo de especies forrajeras por otras menos valiosas como consecuencia del sobrepastoreo bovino.

Estas áreas con severas limitaciones climáticas y edáficas se caracterizan por la variación en las precipitaciones entre años y entre estaciones, así durante los períodos lluviosos los productores tienden a aumentar la carga ganadera, mientras que en los secos ésta no se reduce con la misma velocidad y en similar magnitud (Stritzler, 2010). En los últimos 150 años, la fisonomía de los pastizales naturales se ha transformado profundamente, con un continuo avance de la frontera agropecuaria desde el este húmedo hacia el oeste semiárido (De María et al., 2008; Ulrich, 2009). El avance de la agricultura sobre los sistemas ganaderos agravó la situación, desplazando animales hacia zonas marginales (Stritzler, 2010). En la actualidad en estos ambientes encontramos áreas fragmentadas o parches de vegetación natural alternados con pasturas introducidas.

Las estrategias más frecuentes para recuperar la vegetación en áreas degradadas incluyen la siembra de especies forrajeras, tratamientos para mejorar las características del suelo o la creación de micrositios favorables para el establecimiento y el crecimiento de plantas (Quiroga et al., 2009). La siembra e implantación de especies perennes de buena calidad forrajera permite concentrar la carga animal, logrando así el descanso y producción de semillas de los pastizales naturales; y por otro lado, se evita la roturación frecuente de suelos no aptos para ello (Stritzler, 2010). Distintas investigaciones se han enfocado mayormente en la introducción de especies exóticas adaptadas a ambientes semiáridos (Ulrich, 2009) como es el caso del pasto llorón (Eragrostis curvula) y del mijo perenne (Panicum coloratum) entre otras (Petruzzi et al., 2003; Covas, 1991). Este reemplazo del pastizal por especies exóticas puede significar una solución eficaz desde el punto de vista productivo, ante la falta de forraje. Sin embargo, es necesario incrementar la investigación y conservación de forrajeras nativas a fin de no provocar un mayor deterioro del ambiente perjudicando la biodiversidad.

Trichloris crinita es una gramínea forrajera, nativa, perenne, de ciclo estival, conocida como pasto de hoja, plumerito (Cano, 1988) o cola de caballo (Rúgolo de Agrasar et al., 2005). Con respecto a su fenología, ésta rebrota a fines de Octubre y principios de noviembre, florece y fructifica desde diciembre hasta abril y se caracteriza porque se seca con las fuertes heladas de otoño (Cano, 1988). Trichloris crinita crece en suelos de textura fina, secos o salinos, es común en el Caldenal (Prosopis caldenia Burk.) y alrededor de salitrales, y también se la puede encontrar en algunos arbustales de ja- rilla (Larrea divaricada Cav.) (Cano, 1988). Es una especie de amplia distribución en el continente americano: sur de EEUU, México, Paraguay, Bolivia, Argentina, Chile y Uruguay (Cano, 1988; Cavagnaro et al., 2006) que ha evolucionado en diferentes ambientes y por lo tanto es factible encontrar adaptaciones locales y diferencias en caracteres de importancia agronómica entre las poblaciones (Greco et al., 2002). En Argentina se la encuentra formando parte de los pastizales áridos y semiáridos, y su domesticación y resiembra permitiría mejorar la disponibilidad de forraje en los mismos para consumo directo por parte de ganado (Cano, 1988).

La variabilidad de caracteres dentro de individuos de una especie es la base para los procesos de selección natural y mejoramiento genético por el hombre. Las características morfológicas de las plantas forrajeras poseen un papel clave en su capacidad para adquirir recursos, en su habilidad competitiva y en su interacción con herbívoros (Quiroga, 2011). Se ha evaluado la diversidad genética de T. crinita en América del Sur, utilizando marcadores AFLP para determinados caracteres morfológicos y rasgos agronómicos cuantitativos (Cavagnaro et al., 2006). Además, se han realizado evaluaciones de su comportamiento agronómico en las provincias argentinas de La Pampa (Frank et al., 1990), San Luis (Gabutti et al., 2011), La Rioja y Catamarca (Quiroga, 2011). En dichos trabajos se encontraron valores de producción de biomasa muy variables (200-800 $\mathrm{g} / \mathrm{m}^{2}$ ), aprovechándose su forraje verde en primavera y verano, y también siendo factible su utilización como diferido durante el invierno (Cano, 1988).

El objetivo de este trabajo fue caracterizar morfológicamente y evaluar la biomasa aérea, la calidad de la misma y la persistencia bajo cortes de diferentes poblaciones de Trichloris crinita pertenecientes a Argentina, de las regiones del Caldenal (con predominio de Prosopis caldenia), del Monte (con predominio de arbustos) y del ecotono entre ambos.

\section{MATERIAL Y MÉTODOS}

El trabajo se realizó con 13 poblaciones de Trichloris crinita provenientes de la región del Monte (la cual se extiende desde el NO al centro de Argentina): provincias de Catamarca (1 a 5) y La Rioja (6 a 11), del Caldenal (zona central de Argentina): provincia de La Pampa (12) y del ecotono entre ambos (13) (Cabrera, 1976).

Las semillas fueron colectadas y trilladas manualmente en verano de 2010, posteriormente se almacenaron en sobres de papel hasta la siembra. Estas provenían de jardines de introducción en las provincias de Catamarca, La Rioja y La Pampa. La siembra se realizó el día 5 de Noviembre de 2010, sin ningún tratamiento previo. Dicha actividad se llevó a cabo en invernáculo, en bandejas de germinación con celdas separadas de aproximadamente $3 \mathrm{~cm}$ de diámetro y $5 \mathrm{~cm}$ de alto (speedling), que contenían tierra (suelo de textura franco arenosa del Caldenal pampeano) y arena en proporción 1:2 respectivamente. En cada celda se sembraron 3 semillas (antecio fértil: cariopsis+glumelas acompañado de 2 antecios estériles). Se realizó un seguimiento semanal del número de plantas emergidas de cada población. Las bandejas fueron regadas periódicamente con 
agua destilada. A los 20 días se comenzó con el conteo del número de hojas/planta. Cuando las plantas alcanzaron el estado de 4-5 hojas se colocaron las bandejas de germinación fuera del invernáculo, protegidas con media sombra, por aproximadamente dos meses para la aclimatación o rustificación de las plantas.

A los 105 días de la siembra, se efectuó el trasplante a campo, en INTA EEA Anguil Ing. Agr. Guillermo Covas (longitud: $64^{\circ} 00^{\prime}$, latitud $36^{\circ} 31^{\prime}$ ), La Pampa (Argentina) en un suelo haplustol éntico, de textura franca arenosa, cuyo contenido de Fósforo y Nitrógeno total en la capa arable $(0,20 \mathrm{~cm})$ arrojó valores de 33,59 ppm y $0,15 \%$, respectivamente, mientras que el $\mathrm{pH}$ fue de 5,59. Los valores de $\mathrm{N}$ y $\mathrm{P}$ son considerados altos, mientras que el $\mathrm{pH}$ clasifica al suelo como ligeramente ácido. Las precipitaciones anuales para el período del ensayo fueron: $751 \mathrm{~mm}$ (2011), $884 \mathrm{~mm}$ (2012) y 476 mm (2013), mientras que la media histórica es de 759 $\mathrm{mm}$. En la figura 1 se muestra el diagrama ombrotérmico construido con datos históricos desde 1973 provenientes de la Estación Meteorológica INTA Anguil, La Pampa, Argentina.

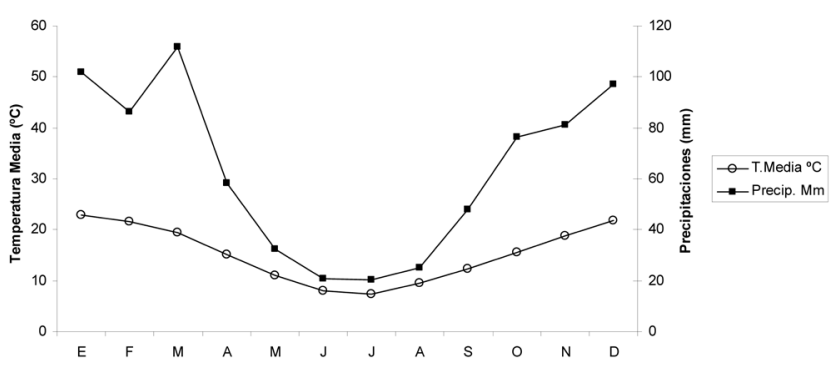

Figura 1. Diagrama ombrotérmico correspondiente a la localidad de Anguil, La Pampa, Argentina. Realizado con valores medios de precipitación y temperaturas desde 1973 (Ombrothermic diagram corresponding to the town of Anguil, La Pampa, Argentina. Made with mean values of precipitation and temperatures from 1973).
El ensayo a campo consistió en bloques al azar con tres repeticiones (tabla I). Las parcelas fueron de 4 líneas con 2 plantas cada una, separadas $50 \mathrm{~cm}$ entre sí; la distancia entre parcelas fue de $50 \mathrm{~cm}$ y cada parcela sirvió de bordura de la contigua; alrededor de todo el ensayo se plantó una bordura de un surco con igual distancia entre plantas que en las parcelas. La unidad de muestreo fueron las 4 plantas centrales de la parcela.

Se efectuaron dos cortes de biomasa acumulada al año, durante 2012 y 2013, con intervalos de 1329, 1373 y 1134 grados días. El corte de otoño, después de la primera helada, se realizó con el objetivo de determinar la biomasa total producida durante el verano y el de comienzos del verano para evaluar la biomasa total producida desde el rebrote primaveral, ambos simulando forraje disponible para el pastoreo. Con la finalidad de evaluar cuales poblaciones presentaban mayor crecimiento inicial, durante el año de implantación (2 y 14 de diciembre de 2011 y 23 de marzo de 2012), sobre las cuatro plantas centrales de cada parcela, se realizaron determinaciones de número de panojas por planta, altura medida hasta la panoja más alta, altura del dosel. El número de macollas por planta se determinaron el 14 de diciembre de 2011 (a los 9 meses de la implantación) y el 15 de mayo de 2012 donde también se determinó diámetro de mata. Además se observaron características morfológicas de la planta como: porte (postrado, erecto, semierecto), presencia de estolones, color de vaina y de panojas, pilosidad y ancho de hoja. Una vez cortadas las plantas se pesaron en verde (peso fresco) y se extrajo una muestra de 100-200 g que se secó en estufa $\left(60^{\circ} \mathrm{C}\right)$ hasta peso constante para el cálculo de porcentaje de materia seca y determinación de peso seco (g MS/planta), considerando solamente las plantas vivas al realizar el cálculo. En el primer corte se guardó una alícuota para efectuar análisis de proteína bruta (PB; AOAC, 1990), digestibilidad de la materia seca estimada a partir de fibra detergente ácida (DMS= 88,9-(0,779*FDA), \% en base seca, y fibra detergente neutro (FDN) (Goering y Van Soest, 1970).

Tabla I. Biomasa media (gr/planta, corresponde a una superficie de 0,25 $\mathrm{m}^{2}$ ) de cortes efectuados en otoño (1) y principios del verano (2) de dos temporadas de crecimiento y persistencia (\% de plantas vivas) al final del período evaluado (Mean biomass ( $\mathrm{g} /$ plant, corresponds to an area of $0,25 \mathrm{~m}^{2}$ ) of cuts made in autumn (1) and early summer (2) two growing seasons and persistence (\% of live plants) at the end of the evaluated period).

\begin{tabular}{|c|c|c|c|c|c|}
\hline \multirow{2}{*}{ Población } & \multicolumn{4}{|c|}{ Peso seco (gr/planta) } & \multirow{2}{*}{$\begin{array}{c}\text { Persistencia } \\
(\%)\end{array}$} \\
\hline & $27 / 04 / 12(1)$ & $09 / 01 / 13(2)$ & $17 / 05 / 13(1)$ & $27 / 12 / 13(2)$ & \\
\hline 1 & $53^{c}$ & $79,35^{\text {bc }}$ & $163,54^{\mathrm{a}}$ & $49,33^{c}$ & $87^{a}$ \\
\hline 2 & $33,33^{c}$ & $60,11^{\mathrm{bcb}}$ & $162,56^{a}$ & $48,67^{c}$ & $82^{\mathrm{ab}}$ \\
\hline 3 & $74,33^{\mathrm{bc}}$ & $88,84^{a b c}$ & $169,17^{a}$ & $73,52^{\mathrm{bc}}$ & $60^{\mathrm{bc}}$ \\
\hline 4 & $60 b^{c}$ & $86,21^{a b c}$ & $93,08^{b c}$ & $59,33^{b c}$ & $87^{a}$ \\
\hline 5 & $112,67^{a}$ & $89,31^{\mathrm{ab}}$ & $165,83^{a}$ & $70,78^{\mathrm{abc}}$ & $89^{a}$ \\
\hline 6 & $60 b^{c}$ & $67,89^{\mathrm{abcd}}$ & $125,02^{\mathrm{abc}}$ & $64^{\mathrm{bc}}$ & $77^{\mathrm{abc}}$ \\
\hline 7 & $31,33^{c}$ & $25,33^{d}$ & $87,26^{c}$ & $17,02^{\mathrm{d}}$ & $10^{d}$ \\
\hline 8 & $47^{\mathrm{bc}}$ & $83,03^{a b c}$ & $154,04^{\mathrm{ab}}$ & $84,01^{\mathrm{ab}}$ & $86^{a}$ \\
\hline 9 & $56,34^{\mathrm{bc}}$ & $83,79^{a b c}$ & $108,22^{\mathrm{abc}}$ & $56,02^{c}$ & $79^{\mathrm{abc}}$ \\
\hline 10 & $89,68^{\mathrm{ab}}$ & $112,23^{\mathrm{a}}$ & $161,02^{\mathrm{a}}$ & $92^{\mathrm{a}}$ & $58^{c}$ \\
\hline 11 & $47,32^{\mathrm{bc}}$ & $47,27^{\mathrm{bcd}}$ & $112,49^{\mathrm{abc}}$ & $58,66^{\mathrm{bc}}$ & $92^{\mathrm{a}}$ \\
\hline 12 & $39^{b c}$ & $41,74^{\mathrm{cd}}$ & $63,98^{c}$ & $60,35^{\mathrm{bc}}$ & $83^{a}$ \\
\hline 13 & $36,33^{c}$ & $79,03^{a b c}$ & $76,13^{c}$ & $73,67^{a b c}$ & $95^{a}$ \\
\hline
\end{tabular}

Letras iguales en una columna indican que no hay diferencias significativas (LSD, $p<0,05)$. 
Las diferencias entre tratamientos fueron evaluadas mediante el análisis de la varianza y la separación de medias por la prueba de diferencias mínimas significativas (LSD, p<0,05), utilizando el paquete estadístico Infostat versión 2002. También se llevó a cabo un análisis de correlación de Pearson.

\section{RESULTADOS}

\section{EMERGENCIA EN INVERNÁCULO}

A los 4 días de la siembra (d.d.s.) se pudo observar que las poblaciones que tuvieron una emergencia más rápida fueron las provenientes de La Rioja: 9 (77\%) seguidas por la 8 y la 10 (ambas $60 \%$ ). Por el contrario, la 3 presentó el menor porcentaje de emergencia (11\%). La población 12 fue la que tuvo mayor emergencia (59\%), mientras que la 13 presentó un $18 \%$. A los 25 y
45 d.d.s., todas las poblaciones tenían una emergencia entre el 50 y $70 \%$, salvo la 7 , que a pesar de que aumentó su porcentaje, siguió manteniéndose en niveles bajos comparada con las demás. También se realizó el conteo de hojas por plántula y se pudo determinar que a los 35 d.d.s, aproximadamente el $60 \%$ del total de plántulas emergidas presentaron entre 5 y 6 hojas.

\section{DETERMINACIONES A CAMPO}

En la tabla II se muestran los resultados de biomasa aérea total en gramos de materia seca por planta obtenidos en cada población, correspondientes a las fechas de corte. Considerando los cuatro periodos de acumulación de biomasa evaluados (correspondientes a dos períodos de crecimiento continuo), se puede observar que la población que presentó mayor biomasa aérea fue la 5 de Catamarca, la cual no mostró diferencias significativas con respecto a la población 10 de La Rioja.

Tabla II. Altura (cm) a nivel del dosel (AD), a nivel de la panoja más alta (AP) y número de panojas por planta de poblaciones de Trichloris crinita hasta el primer año luego del transplante a campo (Height (cm) at the canopy level (AD), at the level of the highest panicle (AP) and number of panicles per plant populations Trichloris crinita to the first year after transplantation to field).

\begin{tabular}{|c|c|c|c|c|c|c|c|c|c|}
\hline \multirow{2}{*}{ Población } & \multicolumn{3}{|c|}{ 02-dic-11 } & \multicolumn{3}{|c|}{ 14-dic-11 } & \multicolumn{3}{|c|}{ 23-mar-12 } \\
\hline & $\mathrm{AD}(\mathrm{ns})$ & AP & $\mathrm{N}^{\circ}$ panojas & $\mathrm{AD}(\mathrm{ns})$ & AP & $\mathrm{N}^{\circ}$ panojas & AD (ns) & $\mathrm{AP}$ & $\mathrm{N}^{\circ}$ panojas \\
\hline 1 & 25,7 & $57,6^{c}$ & $0,53^{\mathrm{cd}}$ & 27,3 & $70,3^{\mathrm{bc}}$ & $1,72^{c}$ & $30,5^{\mathrm{ab}}$ & $96,8^{a}$ & $26,75^{\mathrm{abcd}}$ \\
\hline 2 & 26,7 & - & - & 31,3 & - & 0 & $28,6^{\mathrm{ab}}$ & $89,5^{a}$ & $20,72^{\text {cd }}$ \\
\hline 3 & 21,0 & $25,4^{d}$ & $0,07^{d}$ & 30,0 & $56,0^{\text {bc }}$ & $0,93^{c}$ & $13,6^{b}$ & $41,9^{b}$ & $23^{\text {bcd }}$ \\
\hline 4 & 28,3 & $49,6^{d}$ & $1,25^{\mathrm{cd}}$ & 33,3 & $75,3^{\mathrm{bc}}$ & $2,56^{\mathrm{bc}}$ & $39,5^{a}$ & $94,6^{a}$ & $25,45^{\mathrm{abcd}}$ \\
\hline 5 & 25,0 & $55,3^{c}$ & $0,87^{\mathrm{cd}}$ & 37,0 & $81,7^{b c}$ & $2,41^{b c}$ & $33,7^{a}$ & $91,7^{\mathrm{a}}$ & $36,25^{\mathrm{abc}}$ \\
\hline 6 & 23,0 & $54,6^{c}$ & $0,57^{\mathrm{cd}}$ & 35,0 & $76,7^{\mathrm{bc}}$ & $1,63^{c}$ & $34,7^{\mathrm{a}}$ & $99,1^{\mathrm{a}}$ & $29,1^{\text {abcd }}$ \\
\hline 7 & 20,3 & $38,6^{\mathrm{cd}}$ & $1,81^{b c}$ & 28,3 & $50,67^{c}$ & $4,91^{\mathrm{ab}}$ & $25,8^{\mathrm{ab}}$ & $56,7^{\mathrm{b}}$ & $35,67^{a b c}$ \\
\hline 8 & 20,7 & $45,6^{\mathrm{cd}}$ & $0,25^{\mathrm{cd}}$ & 40,0 & $61,7^{\mathrm{bc}}$ & $1,15^{\mathrm{c}}$ & $32,6^{a}$ & $92,9^{a}$ & $24,47^{\mathrm{abcd}}$ \\
\hline 9 & 20,8 & $48,8^{\mathrm{cd}}$ & $0,38^{\mathrm{cd}}$ & 37,2 & $87,3^{\mathrm{b}}$ & $1,42^{\mathrm{c}}$ & $33,8^{a}$ & $105,1^{\mathrm{a}}$ & $15,64^{d}$ \\
\hline 10 & 24,7 & $56,9^{c}$ & $0,93^{\mathrm{cd}}$ & 30,7 & $77,3^{\text {bc }}$ & $2,46^{\mathrm{bc}}$ & $38,1^{a}$ & $97,9^{a}$ & $39,90^{\mathrm{a}}$ \\
\hline 11 & 19,7 & - & - & 26,7 & $58,7^{\mathrm{bc}}$ & $0,31^{c}$ & $33,1^{a}$ & $92,0^{\mathrm{a}}$ & $23,48^{\mathrm{bcd}}$ \\
\hline 12 & 25,3 & $57,7^{c}$ & $3,94^{a}$ & 28,3 & $66,3^{\text {bc }}$ & $6,25^{a}$ & $29,5^{\mathrm{ab}}$ & $85,2^{a}$ & $37,99^{a b}$ \\
\hline 13 & 18,7 & $54,5^{c}$ & $2,85^{\mathrm{ab}}$ & 27,0 & $79,0^{\text {bc }}$ & $7,57^{a}$ & $29,1^{\mathrm{ab}}$ & $94,6^{a}$ & $25,04^{a b c}$ \\
\hline
\end{tabular}

$\mathrm{AD}=$ altura dosel; $\mathrm{AP}=$ altura panoja; $\mathrm{N}^{\circ}$ panojas= número de panojas. En una misma columna, letras iguales indican que no hay diferencias significativas entre poblaciones (LSD, $p<0,05)$; $n s=$ no significativo. Se muestran valores medios por parcela (cuatro plantas por parcela).

Tabla III. Porcentaje de materia seca (MS), proteína bruta (PB), fibra detergente neutro (FDN) y digestibilidad de la materia seca estimada a partir de fibra detergente ácida (DMS) de 13 poblaciones de Trichloris crinita de Argentina. Datos correspondientes al corte del 27 de abril de 2012 (Percentage of dry matter (DM), crude protein (CP), neutral detergent fiber (NDF) digestibility and estimated from acid detergent fiber (DMS) of 13 populations of Argentina Trichloris crinita dry matter. Data corresponding to court April 27, 2012).

\begin{tabular}{|c|c|c|c|c|}
\hline Población & MS (\%) & PB (\%) & FDN (\%) & DMS (\%) \\
\hline 1 & $41,41^{\mathrm{bcd}}$ & $7,43^{\text {de }}$ & $76,60^{\text {bcdef }}$ & $51,74^{\mathrm{bcd}}$ \\
\hline 2 & $43,15^{\mathrm{abc}}$ & $7,97^{\text {bcde }}$ & $78,28^{\mathrm{abcd}}$ & $51,94^{\mathrm{bcd}}$ \\
\hline 3 & $41,14^{\mathrm{bcd}}$ & $8,36^{\mathrm{bc}}$ & $75,42^{\text {ef }}$ & $54,28^{a b c}$ \\
\hline 4 & $46,11^{\mathrm{ab}}$ & $7,60^{\text {cde }}$ & $79,03^{\mathrm{ab}}$ & $53,09^{a b c d}$ \\
\hline 5 & $37,21^{d}$ & $8,75^{\mathrm{ab}}$ & $75,08^{f}$ & $54,42^{\mathrm{ab}}$ \\
\hline 6 & $41,90^{\mathrm{bcd}}$ & $8,09^{\text {bcde }}$ & $76,99^{\text {bcdef }}$ & $57,24^{a}$ \\
\hline 7 & $45,84^{\mathrm{ab}}$ & $9,44^{a}$ & $74,87^{f}$ & $54,74^{\mathrm{ab}}$ \\
\hline 8 & $43,55^{\mathrm{abc}}$ & $7,58^{\text {cde }}$ & $78,74^{\mathrm{abc}}$ & $51,08^{\text {bcd }}$ \\
\hline 9 & $40,40^{\mathrm{bcd}}$ & $7,70^{\text {cde }}$ & $76,37^{\text {cdef }}$ & $54,53^{a b}$ \\
\hline 10 & $40,78^{\mathrm{bcd}}$ & $8,17^{\text {bcde }}$ & $76,01^{\text {def }}$ & $54,26^{\mathrm{abc}}$ \\
\hline 11 & $39,90^{c d}$ & $8,32^{\mathrm{bcd}}$ & $75,78^{\text {def }}$ & $52,80^{\text {bcd }}$ \\
\hline 12 & $48,07^{a}$ & $7,88^{\text {bcde }}$ & $77,77^{\text {abcde }}$ & $50,15^{\mathrm{cd}}$ \\
\hline 13 & $48,80^{\mathrm{a}}$ & 7,29 & $80,07^{a}$ & $49,79^{d}$ \\
\hline
\end{tabular}

Letras iguales en una columna indican que no hay diferencias significativas (LSD, $p<0,05)$. 
Por otro lado, la que presentó un menor rendimiento fue la 7 de La Rioja. La población del Caldenal no se diferenció significativamente de la del Ecotono presentando rendimientos intermedios. También se muestra en la tabla II que la población de menor persistencia al cabo de las dos temporadas de cortes fue la 7 con apenas $10 \%$ de las plantas vivas seguida por la población 10 con $58 \%$.

El número de panojas por planta es un dato importante a tener en cuenta para la producción de semilla, y por ende para la difusión de un cultivar. La tabla III presenta los datos referentes al número de panojas por planta que registró cada población, en tres fechas diferentes correspondientes a verano y otoño, reflejando una amplitud del período reproductivo que se prolonga durante todo el verano. Comparando la primera determinación con la última, el número de panojas fue aumentando considerablemente con la edad de las plantas, las poblaciones 12 y 13 fueron las más precoces presentando la mayor cantidad de panojas en diciembre del 2011; mientras que las poblaciones 2 y 11 en ese momento aun no estaban panojadas. Sin embargo en otoño son igualadas por otras poblaciones (5, 7 y 10). Es importante destacar la población 6 (Chamical INTA), ya que la misma ha sido incluida en el Registro Nacional de Cultivares (Legajo No 12645). Dicha población presentó valores intermedios en cuanto al número de panojas por planta y a la producción de biomasa. Por otro lado, se realizaron observaciones de las panojas que se fueron cosechando periódicamente, se midió el largo de las mismas, registrándose una longitud máxima de $12 \mathrm{~cm}$ (población 8). Dentro de las poblaciones que presentaron panojas con menor longitud $(9 \mathrm{~cm})$ se incluyeron las líneas 12 y 13. También se pudieron diferenciar aquellas panojas con aspecto plumoso como es el caso de la población 3 y panojas de coloración más oscura o bordó (población 8), comparada con otras como las del Caldenal que son de color claro.

En general, las plantas crecieron en altura durante el transcurso del ensayo, alcanzando en otoño, hasta un metro, sin diferencias significativas entre poblaciones, salvo la 3 y 7 , que en todas las fechas presentaron alturas inferiores a los $60 \mathrm{~cm}$ (tabla III). En la misma tabla se muestran las alturas del dosel registradas en cada

\begin{tabular}{l|ccccccccccccc}
\hline III & 11 & 1 & 5 & 3 & 12 & 6 & 4 & 13 & 7 & 10 & 2 & 9 & 8 \\
II & 13 & 10 & 11 & 9 & 1 & 8 & 2 & 7 & 3 & 6 & 4 & 12 & 5 \\
I & 2 & 1 & 12 & 6 & 9 & 10 & 13 & 4 & 8 & 3 & 5 & 7 & 11 \\
\hline
\end{tabular}

Figura 2. Diseño experimental. Plano del ensayo a campo: Bloques al azar con 3 repeticiones, parcelas con 8 plantas cada una (Experimental design. Plane field trial: randomized block design with 3 replications, plots with 8 plants each).

población en las mismas tres fechas que la variable precedente. Las dos primeras fechas presentaron diferencias no significativas. En otoño de 2012 se observó que las poblaciones de mayor altura de dosel $(\mathrm{p}<0,05)$ fueron la 4, 5, 6, 8, 9, 10 y 11. Esto se puede relacionar con la producción de biomasa que en general coincide, ya que las mayores biomasas correspondieron principalmente a las poblaciones 5 y 10 . Caso contrario a la población 3, que presentó una menor altura del dosel y una elevada producción de biomasa. Esto puede ser explicado teniendo en cuenta características morfológicas, ya que en dicha población se observaron plantas semi-erectas y con tendencia a desarrollar estolones.

El diámetro de la mata está representado en la figura 2 donde las poblaciones más representativas fueron la 5, 10 y 11. La población 7 (La Rioja), presentó el menor diámetro, y en general, en todas las variables evaluadas, valores bajos, con menor crecimiento y rendimiento potencial, lo cual puede deberse a que la misma es proveniente de ambientes salinos y está adaptada al estrés.

El número de macollas por planta se determinó en diciembre de 2011 y mayo de 2012. En la figura 3, para la primera fecha se puede observar que la población con mayor presencia de macollas por planta fue la 5 , que en general tuvo valores elevados en todas las variables evaluadas. Aquellas poblaciones que presentaron un menor número de macollas fueron la 2 y la 11. En otoño de 2012, se observó en todas las poblaciones, un aumento en el número de macollas, donde la población 10 fue la más representativa, mientras que la 2 y 7 presentaron la menor cantidad.

El análisis de correlación de Pearson (n:39) mostró que la biomasa aérea se correlacionó significativamente con el número de macollas/planta $(\mathrm{r}=0,57 ; \mathrm{p}<0,01)$,

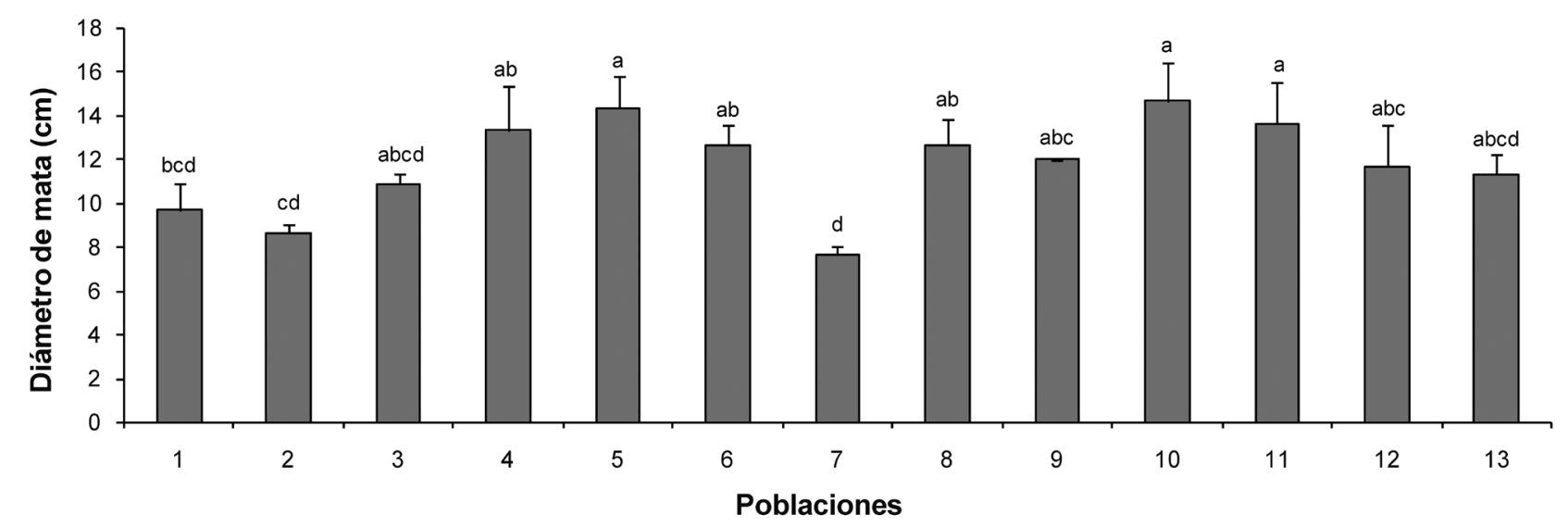

Figura 3. Diámetro de mata luego del primer corte (mayo 2012) en poblaciones de T. crinita de Argentina. Letras distintas indican diferencias significativas (DMS, $\mathrm{p}<0,05)$ (Diameter then kills the first cut (May 2012) in populations of T. crinita of Argentina. Different letters indicate significant differences (LSD, $p<0.05)$ ). 
con el diámetro de mata $(\mathrm{r}=0,64 ; \mathrm{p}<0,01)$, número de panojas $(\mathrm{r}=0,38 ; \mathrm{p}<0,02)$ y altura hasta la panoja $(r=0,35 ; p<0,04)$. Sin embargo, no hubo correlación significativa entre biomasa y las variables de calidad del forraje (PB, DMS, FDN).

Las poblaciones de la región del Caldenal y del Ecotono fueron en general las que presentaron mayor porcentaje de MS, bajo porcentaje de proteína, mayor FDN y menor DMS (figura 4). Por el contrario, la población 7 mostró un elevado porcentaje de proteína y por lo tanto, menor contenido de fibra. Esto puede deberse a que las plantas de dicha población presentaron tallos finos y mayor altura de dosel en relación a la altura de las panojas, es decir, mayor proporción de hojas en su parte aérea.

\section{DISCUSIÓN}

La variabilidad de caracteres dentro de individuos de una especie es la base para los procesos de selección natural y mejoramiento genético por el hombre. Las características morfológicas de las plantas forrajeras poseen un papel clave en su capacidad de adquirir recursos, en su habilidad competitiva y en su interacción con herbívoros (Quiroga, 2011).

Los resultados de este estudio indican diferencias significativas en diversas variables fenotípicas y de interés agronómico, y son coincidentes con los realizados por Gabutti et al. (2011) quienes evaluaron características agronómicas en poblaciones de Trichloris crinita y Trichloris pluriflora provenientes de las provincias de La Rioja, Catamarca, Mendoza, San Juan, San Luis y Córdoba (Argentina). Quiroga (2011) trabajando con poblaciones provenientes de Catamarca, La Rioja y Córdoba (Argentina), también encontró diferencias fenotípicas para variables como altura de dosel, número de macollas y hábito de crecimiento o porte.

Las producciones de forraje fueron muy variables, encontrándose un valor máximo de $664 \mathrm{~g} \mathrm{MS} / \mathrm{m}^{2}$ y un valor mínimo de $68 \mathrm{~g}$ MS $/ \mathrm{m}^{2}$ para las poblaciones $5 \mathrm{y}$ 7 respectivamente. En estudios realizados en La Rioja (Namur et al., 2011), evaluaron la disponibilidad hídrica como el principal control de la producción forrajera, encontrándose una producción de forraje entre 799 y
$237 \mathrm{~g}$ MS $/ \mathrm{m}^{2}$. Mientras que en Tucumán (Argentina), Díaz et al. (1970), encontraron rindes de $324 \mathrm{~g} \mathrm{MS} / \mathrm{m}^{2}$. Por otro lado, Echavarría (1973) también determinó la producción de forraje en esta especie en México, totalizando entre 270 y $500 \mathrm{~g} \mathrm{MS} / \mathrm{m}^{2}$. En general las 2 poblaciones que presentaron mayor biomasa total tanto en otoño como en primavera fueron la 5 y 10, mientras que las poblaciones 7 y 12 estuvieron entre las menos productivas (tabla II). En un estudio realizado por Frank et al. (1990) referido a la productividad de los pastizales del sector Meridional de la provincia de La Pampa, con una precipitación media anual de 350 a $400 \mathrm{~mm}$ y suelo de textura franco-arenosa, la especie estival que aportó la mayor cantidad de forraje fue Trichloris crinita (64 kg MS/ha), comparada con otras especies, tales como Aristida mendocina y Sporobolus cryptandrus, cuyos aportes fueron menores.

Algunos autores han señalado que es necesario estudiar la calidad del forraje y persistencia bajo pastoreo para definir cual especie y población presenta mayor aptitud forrajera (Gabutti et al., 2011). Respecto a Trichloris crinita, Cano (1988) indicó que la misma es una especie muy apetecida por el ganado en verano, y además, proporciona buena cantidad y calidad de forraje aun diferida.

La proteína bruta (PB) presentó en general, valores superiores al $7 \%$ (requerimiento de una vaca de cría), coincidiendo con los de Wainstein y González (1971) quienes analizando material proveniente de Mendoza (Argentina) encontraron valores de 6,74 \% de PB. Por otro lado, Abiusso (1964), trabajó con material procedente del Depto. de Loventué- La Pampa (Argentina) obteniendo valores de PB de 9,44 \%. Los mismos fueron ligeramente inferiores a los encontrados por Chirino et al. (1988) para otra especie estival nativa de Argentina, Pappophorum caespitosum, la que presentó valores de PB de aproximadamente el $10 \%$. Lo mismo ocurrió con los resultados obtenidos en un trabajo realizado por Veneciano et al. (1996) para Sorghastrum pellitum, cuyos valores de PB también variaron entre el 7-10\% aproximadamente.

Los valores de fibra detergente neutro (FDN: 72$83 \%$ ) fueron similares a los obtenidos en el mismo área de estudio por Fontana et al. (2013) quienes trabajaron

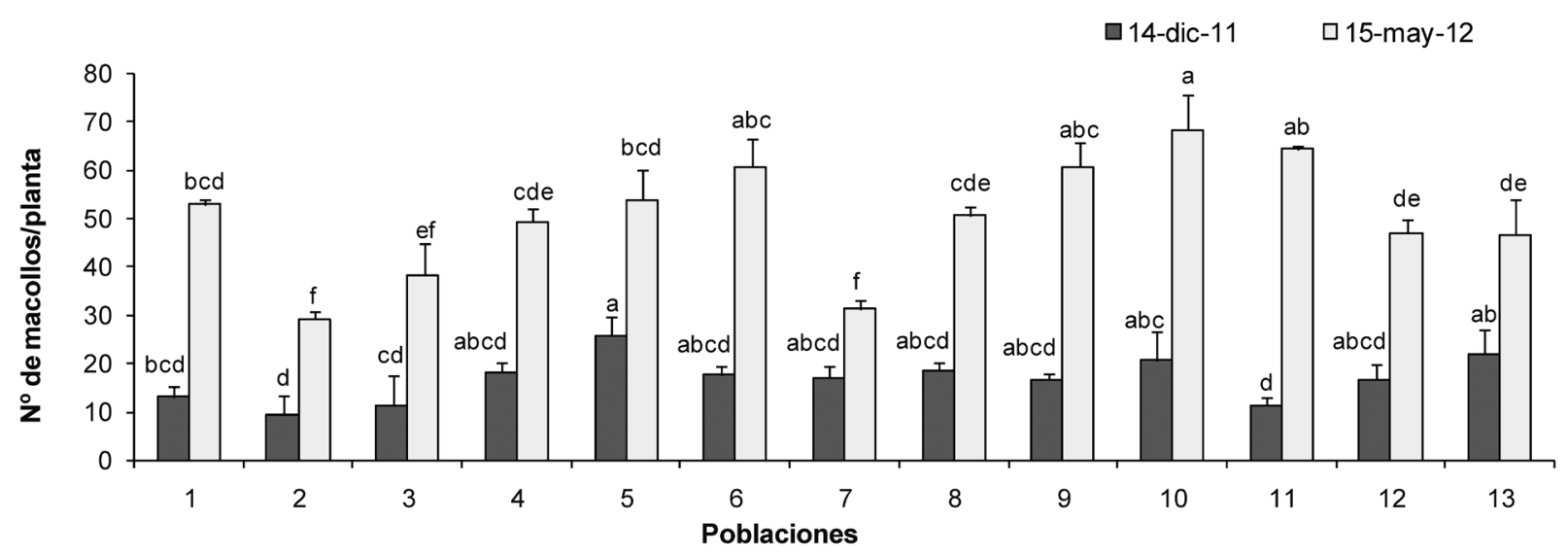

Figura 4. Número de macollas por planta, registrado en dos fechas, en trece poblaciones de T. crinita. Letras distintas indican diferencias significativas (DMS, $\mathrm{p}<0,05)$ (Number of tillers per plant, registered on two dates in thirteen populations of $T$. crinita. Different letters indicate significant differences (LSD, $p<0.05)$ ). 
con forrajeras estivales. En tanto que los valores de PB $(7,4-13 \%)$ fueron en general inferiores a los encontrados para forrajeras introducidas tales como Tetrachne dregei, Panicum coloratum, Digitaria eriantha y Eragrostis curvula. Lo mismo ocurrió con la digestibilidad de la materia seca, que también fueron inferiores a los encontrados en dicho trabajo (DMS: 53-58 \%).

La persistencia de las distintas poblaciones puede variar influenciada por el ambiente. En este trabajo se ha observado que la población proveniente de un bajo salino de La Rioja (7) presentó muerte de gran parte de sus macollas o plantas, a partir del segundo corte realizado el 9 de enero de 2013. Esto podría tener que ver con la altura del corte y con la posición de los brotes. Es por esto que es necesario estudiar la persistencia bajo pastoreo.

En el presente trabajo no se evaluó rendimiento de semilla, pero sí se observó abundante panojamiento especialmente en las poblaciones 12 y 13. Además se ha observado su excelente capacidad para resiembra natural. Considerando que es una especie clave del pastizal de las regiones áridas y semiáridas y que es apta para revegetación de pastizales degradados y áreas desmontadas (Cavagnaro et al., 2006) se deberían intensificar los estudios en producción de semilla con la finalidad de domesticarla y poder sembrarla en campos naturales del Caldenal y del Monte de Argentina, y de esta manera mejorar la condición de los pastizales degradados. Se han realizado experiencias al respecto en Mendoza (Mora et al., 2013) y La Rioja (Blanco et al., 2013) con muy buenos resultados.

\section{CONCLUSIONES}

De acuerdo a los resultados obtenidos, se puede concluir que existe variabilidad entre las poblaciones de Trichloris crinita estudiadas, lo que permitirá seleccionar en función de su importancia agronómica, teniendo en cuenta fundamentalmente la biomasa total, calidad de forraje, producción de semillas y su persistencia bajo corte. En éste trabajo las poblaciones 5 y 10 provenientes de Catamarca y La Rioja, se destacaron respecto de las demás en producción de forraje y la primera mostró elevada persistencia bajo corte; lo cual nos sugiere que podría ser utilizada con la finalidad de mejorar la oferta forrajera de los pastizales de la región semiárida central de la Argentina.

La restauración a partir de la implantación de gramíneas nativas constituiría una alternativa eficiente, ya que las mismas son los principales recursos forrajeros de las regiones áridas y semiáridas, y cuentan con características morfológicas y fisiológicas especiales que les confieren ventajas en este tipo de ambientes actualmente degradados por causas naturales y antrópicas.

\section{AGRADECIMIENTOS}

Este trabajo fue realizado gracias a la ayuda financiera de los proyectos de INTA (PE AEFP 1481: Introducción y Evaluación de cultivares y especies forrajeras, y PAMSL-1282102: Desarrollo territorial sustentable de la región del Caldenal y Oeste árido de la Provincia de La Pampa, Centro Regional la Pampa - San Luis). Se agradece especialmente por la organización de la red de Trichloris crinita en el marco del proyecto nacional mencionado precedentemente, a María del Cármen Spada, Pedro Namur, Emiliano Quiroga y Víctor Hugo Burgui.

\section{BIBLIOGRAFÍA}

Abiusso, N. 1964. Composición química y valor alimenticio de algunas plantas indígenas y cultivadas en la República Argentina (II). Rev Invest Agropec. Ser. 2. Biol Prod Veg, 1: 311-338.

AOAC. 1990. Official methods of analysis (15 th ed.). Association of Official Analytical Chemists. Washington, DC.

Blanco, L.; Namur, P.R.; Ferrando, C.; Rettore, A.; Namur, P.; Ávila, R.; Molina, J. y Orionte, E. 2013. Evolución de la vegetación después del rolado y siembra de pastos nativos en La Rioja. Actas del VI Congreso Nacional de Pastizales, Los pastizales y el hombre. Santa Rosa. La Pampa. Argentina. 125 pp.

Cabrera A. 1976. Regiones fitogeográficas argentinas. En: E.F. Ferreira Sobral (Eds). Enciclopedia Argentina de Agricultura y Ganadería. $2^{a}$ ed. Acme. Buenos Aires. Argentina. p. 90.

Cano, E. 1988. Pastizales naturales de La Pampa. Descripción de las especies más importantes. Tomol. Convenio AACREA-Provincia de La Pampa. Argentina. Editorial AACREA. La Pampa. Argentina. 425 pp.

Cavagnaro P.F.; Cavagnaro, J.B.; Lemes, J.L.; Masuelli, R.W. y Passera, C.B. 2006. Genetic diversity among varieties of the native forage grass Trichloris crinita based on AFLP markers, morphological characters, and quantitative agronomic traits. Genome, 49: 906-918.

Chirino, C.C.; K.M. Norlander Grahny L.E. Robles. 1988. Determinación de proteína bruta de algunas especies forrajeras de La Pampa. Rev Fac Agron, UNLPam. 3: 57-74.

Covas, G. 1991. Introducción del pasto llorón en la República Argentina. In: Fernández, O.A.; R.E. Brévedan y A.C. Gargano. Eds. El pasto llorón. Su biología y manejo. UNS, Bahía Blanca, Bs. As. pp. 1-6.

Demaría, M.; Aguado, S. y Steinaker, D. 2008. Reemplazo y fragmentación de pastizales pampeanos semiáridos en San Luis Argentina. Asociación Argentina de Ecología. Ecol Austral, 18: 55-70.

Díaz, H.B.; Lagomarsino, E.D.; Prete, I.R. y Rodríguez Rey, J.C. 1970. Productividad de las pasturas naturales de las zonas ganaderas de Tucumán. Rev Agron NO Argentino, 7: 675-724.

Díaz, R.O. 2007. Utilización de pastizales naturales. Editorial Encuentro. Córdoba. Argentina. 456 pp.

Echevarría, S.M. 1973. Evaluación del comportamiento de diez especies de zacates nativas o introducidas, sembrados de temporal en la región Central de Chihuahua, México. Pastizales, 4: 2-8.

Fontana, L.M.C.; Ruiz, M.A.; Blain, G.; Babinec, F.J. y Romero, N.A. 2013. Gramíneas $C 4$ con alfalfa en La Pampa semiárida: Producción y calidad de forraje. Rev Argent Prod Anim, 33: 215-305.

Frank, E.O.; Llorens, E.M. y Cabral, D.R. 1990. Productividad de los pastizales de la provincia de La Pampa. EEA INTA Anguil, Subsecretaría de Asuntos Agrarios, Secretaría de Agricultura, Ganadería, Pesca y Alimentos. Cambio Rural. Editorial AACREA. La Pampa. Argentina. 167 pp.

Gabutti, E.G.; Cozzarín, I.G.; Reynoso, M.; Privitello, M.J.L.; Pensiero, J. y Zabala, J.M. 2011 . Caracterización agronómica de poblaciones nativas de Trichoris crinitay T. pluriflora. Rev ArgentProdAnim, 31: 574.

Goering, H.K. and Van Soest, P.J. 1970. Forage fiber analisis (apparatus, regents, procederes and some applications). Agriculture Handbook. USDA. Washington DC. 379 pp.

Greco, S.A. y Cavagnaro, J.B. 2002. Effects of drought in biomass production and allocation in three varieties of Trichloris crinita $P$. (Poaceae) a forage grass from the arid Monte region of Argentina. Plant Ecol, 164: 125-135.

INFOSTAT. 2002. Infostat/Estudiantil, versión 2.0. Grupo Infostat/FCA. Universidad Nacional de Córdoba. Ed. Brujas. Córdoba. Argentina. 
Mora, S.; Cabral, D.y Rosales, I. 2013. Establecimiento de pasto plumerito (Trichloris crinita Parodi) en el año de siembra. Actas del VI Congreso Nacional de Pastizales, Los pastizales y el hombre. Santa Rosa. La Pampa. Argentina. p. 237.

Morici, E.; Ernst, R.; Kin, A.; Estelrich, D.; Mazzola, M. y Poey, S. 2003. Efecto del pastoreo en un pastizal semiárido de Argentina según la distancia a la aguada. Arch Zootec, 52: 59-66.

Morici, E.; Doménech-García, V.; Gómez-Castro, G.; Kin, A.; Sáenz, A. y Rabotnikof, C. 2009. Diferencias estructurales entre parches de pastizal del Caldenal y su influencia sobre el banco de semillas, en la provincia de La Pampa, Argentina. Agrociencia, 43: 529-537.

Namur, P.R.; Blanco, L.J. y Sancho, A.R. 2011. Respuesta forrajera de Trichloris crinita a la disponibilidad hídrica. Rev Argent Prod Anim, 31: 540 .

Nazar Anchorena, J.B. 1988. Pastizales naturales de La Pampa. Manejo en regiones semiáridas. Tomo II. Convenio AACREA - Provincia de La Pampa. Argentina. 112 pp.

Petruzzi, H.J.; Stritzler, N.P.; Adema, E.O.; Ferri, C.M. y Pagella, J.H. 2003. Mijo perenne. Ediciones INTA. Publicación Técnica N 51.28 pp.

Quiroga, E.; Blanco, L. y Orionte, E. 2009. Evaluación de estrategias de rehabilitación de pastizales áridos. Austral Ecol, 19: 107-117.
Quiroga, R.E. 2011. Variación morfológica en once poblaciones del pasto nativo Trichloris crinita. INTA EEA, Catamarca. Argentina. Rev Argent Prod Anim, 31: 539.

Rúgolo de Agrasar, Z.E.; Steibel, P.E. y Troiani, H. O. 2005. Manual ilustrado de las gramíneas de la provincia de La Pampa. Universidad Nacional de La Pampa. Universidad Nacional de Río Cuarto. 191 pp.

Stritzler, N.P. 2010. Producción y calidad de especies forrajeras megatérmicas. Actas de la jornada a campo ¿̇ómo pasar el invierno?: Megatérmicas, disponibilidad forrajera: el uso en diferido. Estancia San Marcos de León. La Pastoril. La Pampa. UE y DT INTA Victorica y UE y DT Inta Villa Mercedes. San Luis. Libro de resúmenes. p. 3.

Ulrich, S.A. 2009. Evaluación de una población de Sorghastrum pellitum (Hack.) Parodi, hasta su reintroducción en áreas medanosas. Tesina Ingeniería en Recursos Naturales y Medio Ambiente. Facultad de Ciencias Exactas y Naturales, Universidad Nacional de La Pampa. Santa Rosa. La Pampa. Argentina. 31 pp.

Veneciano, J.H.; Terenti, O.A.; Sager, R. y Berton, J.A. 1996. Variación estacional de rendimiento, proteína bruta y minerales en Sorghastrum pellitum (Hack.) Parodi (Pasto de vaca). Información Técnica N ${ }^{\circ} 139$, INTA San Luis. 27 pp. 